\title{
A Convenient Verisimilitude or Oppressive Internalization?: Characterizing the Ethical Augments Surrounding Hierarchical Structures in Knowledge Organization Systems
}

\author{
Joseph T. Tennis \\ The Information School of the University of Washington, Box 352840, \\ Mary Gates Hall, Ste 370, Seattle, WA, United States 98195-2840, \\ $<$ jtennis@uw.edu>
}

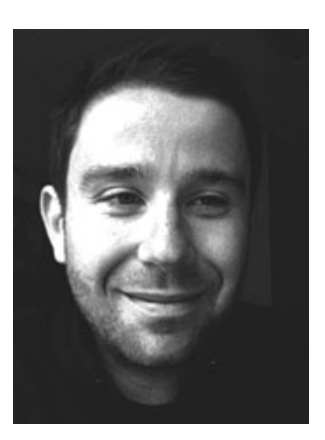

Joseph T. Tennis is an Assistant Professor at the Information School and a member of the textual studies faculty of the University of Washington, an Associate Member of the Peter Wall Institute for Advanced Study at The University of British Columbia, and visiting scholar at the State University of São Paulo. $\mathrm{He}$ is Reviews Editor of Knowledge Organization, Managing Editor for Advances in Classification Research Online, and on the editorial board for The Library Quarterly, member of the Dublin Core Usage Board, and active in the InterPARES research project. He works in classification theory, the versioning of classification schemes and thesauri (a.k.a. subject ontogeny), and the comparative discursive analysis of metadata creation and evaluation, including archival metadata, both contemporary and historical. Ethical Augments Surrounding Hierarchical Structures in Knowledge Organization Systems. Knowledge Organization. 39(5), 394-397. 11 references.

ABSTRACT: This paper considers the ethical concerns that surface around hierarchy as structure in knowledge organization systems. In order to do this, I consider the relationship between semantics and structure and argue for a separation of the two in design and critique of knowledge organization systems. The paper closes with an argument that agency and intention, as ethical concerns in knowledge organization, lead us to argue for a neutral stance on hierarchy.

Received 17 July 2012; Accepted 17 July 2012

\subsection{Introduction}

Hierarchy, broadly defined is a ranking. And though it is applied in many contexts, in the case of bibliographic classification, we are concerned with classes of documents ranked from broad to narrower meaning. There are various ways we can formalize and operationalize this general definition. For example, we might say that broad classes have a greater extension and lesser extension than narrower classes. This would mean there are more items (documents) in a broader class than a narrower class. Likewise, the narrower class would have more characteristics (greater intension) than a broader one. This we will call the functional argument.

If we follow Aitchison, Gilchrist, and Bawden (2000), we are told there are three kinds of hierarchy: whole-part, genus-species, and class-instance. In each of these cases that the same operationalization applies, the whole, genus, and class all have a greater extension and less intension than their subordinates.

However, some have ascribed an interpretation of hierarchy that reads something like this: hierarchy is harmful because it is tool of the dominant paradigm, 
and the dominant paradigm causes harm. For example, there are arguments that hierarchy is a realization of patriarchy, and hence any KOS that uses this structure reflects patriarchal bias (cf., Olson 2007). It can also be said that hierarchy just looks like the power structure of patriarchy, and, as such, should be dismantled to show a form of resistance to patriarchal norms (Daly in cahoots with Caputi 1982). Likewise, we might see the divide-and-control tree diagram of hierarchy schematized as a tool of colonial domination, and so link this structure with settler mentality (Ashcroft, et al. 2001, 23-27). This second group of perspectives we will call emancipation argument.

Reinforcing the interpretation of hierarchy as harmful is the prevalence of the term bierarchical force. This term, in the context of classification research, means that the subordinate class inherits the characteristics of the superclass. For example in a folk classification of animals, we might say that all sparrows are birds. However, the presence of force in this term is evocative of harm to some readers. It is this that makes investigating the differences and similarities that obtain between these two arguments worthwhile.

There are two camps in KO literature with regard to hierarchy and its ethical essence. One combines the semantics and structure of hierarchy into one concern. The other camp separates semantics from structure. The former links the actions of hierarchy described above to the analysis of a domain and the definition of a term. And while this is appropriate in some contexts, in others it is not. We can call the first camp the mixed camp-where semantics and structure are mixed. And we can call the other camp the contiguous camp where semantics and structure are separate but touching, and not intermingled.

The mixed camp calls to our attention the way in which we conflate semantics and structure in knowledge organization systems (KOS's). As intentional designers of KOS's, we should be aware of what we conflate. So the mixed camp brings us some insight into our design assumptions. However, it seems to me, that if all KOS's were designed such that we always conflated semantics and structure, specifically that hierarchy exploits semantics for the sake of its structure, then it can be seen to remove agency for those outside of the dominant paradigm to use hierarchy for their needs. That is, the mixed camp might say that it would be immoral for an oppressed population to use hierarchy because it exploits meaning in a decidedly colonial or patriarchal way.

The contiguous camp takes a different stance. It claims that we can separate semantics and structure, and in fact it is desirable from an intentional design perspective to do so. I will advocate for separating semantics and structure. Because I believe in so doing we highlight the agency we have in making ethical KOS's. Agency and intention are the key ethical concerns I have in the context of knowledge organization and classification theory (Tennis 2009, 2011). This is because a KOS is an intervention, and we want the intervention to function well as well as not cause harm. This means we must design, implement, and evaluate with intention, and we must consider whether or not we do harm when we design, implement, and evaluate KOS's. One step forward in considering intention and agency is to be clear about semantics and structure in KOS's.

\subsection{Semantics and structure}

In the context of classification schemes, the examples par excellence of hierarchy, we can often separate structure and semantics. For example, automobiles might be a superclass and Mazda a subclass. Just as easily, we can have automobiles as a superclass and blue cars as a subclass. The structure is the same. In both cases, we have a hierarchical relationship. And in both cases, we added at least one characteristic to the superclass to generate one, of potentially many, subclasses.

We can contrast this with semantics. The difference between Mazda and blue car, as classes, is their definition-how we define, in this context, their meaning. Mazda in this case is "a particular make of car," and blue car is "the set of all those cars that are blue."

There are, of course, intersections between structure and semantics. We know from the hierarchical structure (if it is coherent) that Mazda in this case is the make of the car, not the car company, or the god of wisdom, intelligence, and harmony. We also know that the class blue car does not refer to train cars, but only to automobiles.

In its original conception, hierarchy was used to describe the orders of angels by Pseudo-Dionysius the Areopagite (fl. 5th-6th centuries). This we see from the Oxford English Dictionary. These are listed in order of proximity to God, where Seraphim are the closest and Angels the furthest from God: Seraphim, Cherubim, and Thrones, Dominations, Virtues, and Powers, Principalities, Archangels, and Angels.

In this instance, we can see the concept of rulership or governance that is part of one definition of hierarchy.

In biology, hierarchy groups kinds of organisms by (current or not) theories of kinds. These are often 
contentions and refined based on new data. For example in paleontology, dinosaurs were traditionally classed along the Linnaean system of ranks. However, many paleontologists do not think this is appropriate and prefer a phylogenetic system of classification that does not have ranks. The latter is more flexible to allow for new data.

In both the religious and biological cases, we are trying to show some relationship. In the first, we are concerned with how close an angel is to God. The second, we are concerned with showing descendent relationships of organisms, which it is theorized, come from a common ancestor, but are now differentiated.

These structures are related to what we build into our classification schemes. We want to show relationships, and we want to show how the whole of the collection is interrelated. We have other requirements as well. If we define classification (strictly) as the mutually exclusive, jointly exhaustive, set of hierarchically and systematically arranged classes, then we can see how hierarchy is a design feature. It is meant to do something. Hierarchy in classification schemes is a design feature-an intervention. It is the primary tool for precision and recall in systems that use classification. The rationale for this design system is well rehearsed in the literature. We understand that the broader the class, the higher the recall, the narrower the class, the higher the precision.

If this is the nature of hierarchical structure, we can see how it is distinct, yet connected to semantics, we can see how the meaning of class, its semantics, in a classification scheme is contextualized, but not defined by its structure. Semantics, in this case, is the definition of a class. So we now must ask: what is a definition?

There are two commonly accepted types of definition. We can have definition by intension and definition by extension. Definition by intension is definition through the use of the genus (and differentia) of a term. Definition by extension is definition through the enumeration of every example of that term. For example,

Intension of the UW iSchool Dean: chief academic administrator in charge of the Information School

Extension of the UW iSchool Dean (past and present): Mike Eisenberg and Harry Bruce

With the above example of an intensional definition, we can see we have characteristics that could be put into a hierarchy: (1) Chief Academic Administrator, (2) Information School. Both of these could be subclasses and super-classes. However, the extensional defintion is not so good at providing characteristics. We do not, from this list, get characteristics used and useful for hierarchy.

Yet this is often the only way we can begin to construct intentional definitions, is through extension (gathering examples). And from there, we can interpret (impose?) characteristics we can use to group classes, thereby moving from extensional definitions toward more formalized intensional definitions.

Another example like this is the platypus (Eco 2000). The characteristics of the animal-the features we might use to put it into its place in the tree of life were contradictory to taxonomy at the time. Yet it was an example of what could be, so its existence is what made it its place—a place in the hierarchy.

\subsection{Intention and agency}

We can now close with a discussion of the relationship of hierarchy to intention and agency and answer the question as to whether it is a convenient verisimilitude or an oppressive internalization.

Intention is a focal point of my philosophical stance. As a neopragmatic zen buddhist (Rorty 1982; Harvey 1995), I must acknowledge that I have agency in my work with words (the primary material of both KOS's and my research), that they are useful, and I can shape the world with my words and actions. To this end, a functional argument associated with the contingent camp seems for me to be the only way to examine hierarchical structures. If it is a tool to use by anyone, then we have added to the toolbox of everyone.

This also opens up the possibility of interpreting other forms of structure as tools to be used-perhaps for different purposes. For example, I can imagine many functions for a web of interconnected nodes as outlined in Olson (2007), or "skeins" following Daly and Caputi (1982). However, these structures may not function in the same way.

Further, separating structure from semantics in the functional and contingent stance allows us to closely examine assumed definitions (often left out in many KOS's), and make them more explicit, thereby acting intentionally. This then allows us to better consider whether we are designing ethically.

So, is hierarchy a convenient verisimilitude or oppressive internalization? Neither. Hierarchy neither represents reality (robustly or in shorthand), nor does it in and of itself serve as oppressive internaliza- 
tion. In the first case, we can see many different realities when we analyze the structure and semantics of a domain, and we should be sensitive to those possibilities (cf., Bowker and Star 2001).

In the latter case, we remove agency of the oppressed if we say that hierarchy is not to be used in a functional manner because it is besmirched by the conflation of semantics and structure. If our work is the work of craft (Olson 2001; Tennis 2009), then we must have the appropriate tools to do that work.

\section{References}

Aitchison, Jean, Glichrist, Alan, and Bawden, David. 2000. Thesaurus construction and use. $4^{\text {th }}$ ed. Chicago: Fitzroy Dearborn.

Ashcroft, Bill, Griffiths, Gareth, and Tiffin, Helen. 2001. Binarism. In Ashcroft, Bill, Griffiths, Gareth, and Tiffin, Helen eds., Key concepts in post-colonial studies. London: Routledge, pp. 19-22.

Bower, Geoffrey C., and Star, Susan Leigh. 2001. Sorting things out: classification and its consequences. Cambridge, Mass.: MIT Press.

Daly, Mary, and Caputi, Jane. 1987. Websters' first new intergalactic wickedary of the English language. Boston: Beacon Press.
Eco, Umberto. (2000). Kant and the platypus: essays on language and cognition. Mariner Books.

Harvey, Peter. 1995. Criteria for judging the unwholesomeness of actions in the texts of Theravāda Buddhism. In Journal of Buddhist ethics 2: 140-51.

Olson, Hope A. 2001. Patriarchal structures of subject access and subversive techniques for change. In Canadian journal of information and library science 26n2/3: 1-29.

Olson, Hope A. 2007. How we construct subjects: a feminist analysis. In Library trends 56n2: 509-41.

Rorty, Richard. 1982. Consequences of pragmatism. Minneapolis: University of Minnesota Press.

Tennis, Joseph T. 2009. "Precepts for engaged knowledge organization." Paper presented at The Ethics of Information Organization conference, Milwaukee, Wisconsin, May 22-23, 2009. Video available: http:// www4.uwm.edu/sois/projects/media/player/ ?episode $=08 f 622 \mathrm{c} 5 \mathrm{bda} 04 \mathrm{a} 72929888 \mathrm{ac0d} 499 \mathrm{~b} 60$.

Tennis, Joseph T. 2011. "Precepts for an engaged knowledge organization." Paper presented at Univerisade de Estaudual Paulista, UNESP, September 2011, Marília, Brazil. 